\title{
A Dynamic Model of The Relationship Between Supply and Demand of European Refugees
}

\author{
Wei Jiang \\ School of North China Electric Power University, Baoding 071000, China; \\ 727105937@qq.com
}

Keywords: refugee crisis, Single-objective optimization, Least square method

Abstract. We take Germany as an example to derive a dynamic model which describes the relation between supply and demand. Through the data we apply least square method to fit the demand changing over time, as well as single objective optimization and radiation network to acquire the mechanism of distribution and transportation of the resources to refugees.

\section{Demand}

Through the statistics we've found, the asylum applications to Europe in 2014 is acquired. The number of applications is shown in Table 1 and the trend is shown in Figure 1.

Table 1. The number of asylum application in Europe

\begin{tabular}{ccccccc}
\hline month & 1 & 2 & 3 & 4 & 5 & 6 \\
\hline number & 37360 & 79550 & 100350 & 186870 & 239380 & 351310 \\
\hline month & 7 & 8 & 9 & 10 & 11 & 12 \\
\hline number & 415960 & 450200 & 465230 & 493090 & 489940 & 510930 \\
\hline
\end{tabular}

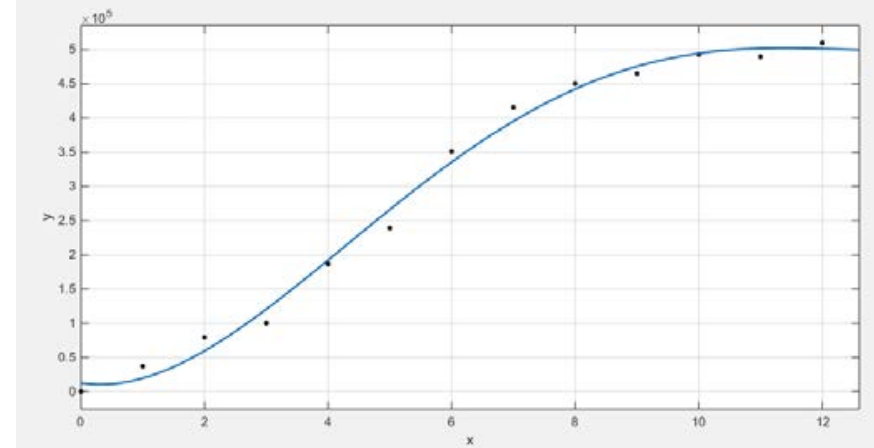

Fig.1 Fitting curve of trend

Least square method is applied to fit the number varying with time. Through the method, we acquire the growth speed of number, allowing us to predict the number in the future and draw the curve of demand.The fitting equation to be

$$
f(\mathrm{t})=p_{1} \cdot \mathrm{t}-p_{2}
$$

Where $p_{1}=4.564 \mathrm{e}+04, p_{2}=-4.256 \mathrm{e}+04$.

We generally assume the fitting curve to be suitable for major countries in Europe including Germany for they enjoy a similar situation when it comes to refugee crisis. The demand in Germany is assured and quantized.

\section{Supply}

We come up with a kind of deployment mechanism that the supplies within a certain range can transport across supplement points. Hubs of settlement points and supplement points were chosen to build the Radiation Network. The network would not only ease the pressure of the transportation and ensure the supplies and demand control due to the presence of hubs. This model ensure the orderly conduct of the resettlement work. 
Two models are included in our mechanism, the distribution of supplies and the dispatch and transportation of different settlement points. The major factor in model of distribution is the total cost, including alterable part and fixed part. On the contrary, the model of transportation barely consider the cost and reflect the weak economy quality.

The parameters and variables of the two models are shown in Table 2.

Table 2 Model Parameters

\begin{tabular}{|c|c|}
\hline Parameter & Meaning \\
\hline $\boldsymbol{I}$ & The set of supplement points \\
\hline $\mathbf{I}$ & The set of settlement points \\
\hline$J$ & The set of resources \\
\hline $\boldsymbol{K}$ & Cost \\
\hline C & Quantity \\
\hline$Q$ & Velocity \\
\hline $\boldsymbol{V}$ & Capacity \\
\hline & Distance \\
\hline$S$ & 0-1 variable ( = 1 when i choose $k$ ) \\
\hline $\boldsymbol{D}$ & 0 - 1 variable ( $=1$ when i choose at least one point) \\
\hline $\boldsymbol{R}_{i k}$ & 0-1 variable ( = 1 when $\mathrm{i}$ choose $\mathrm{j}$ ) \\
\hline \multicolumn{2}{|l|}{$P_{i}$} \\
\hline \multicolumn{2}{|l|}{$T_{i j k}$} \\
\hline SET & The set of supplement points and settlement points \\
\hline & The set of hubs \\
\hline IV hubs & $0-1$ variable ( $=1$ when $i$ is the hub) \\
\hline$h u b_{i}$ & $0-1$ variable ( $=1$ when through hub $\mathrm{m}$ or $\mathrm{n}$ ) \\
\hline flow $_{i j m n}$ & \\
\hline
\end{tabular}

\subsection{Model of distribution}

Single objective optimization is applied in our model of distribution, which is dynamic. The environmental factors including water, food and resources to feed and house the moving migration are changing over time. We generally assume that:

1. The countries' capacity to hold refugees and provide resources can be predicted through the model we have created, the requirement is also acceptable;

2. Cities with supplement points have been chosen;

3. Resources in reserve are limited.

The object owns five type of cost, the total cost of distribution, the alterable cost of resources, transportation cost, delayed cost and loss cost.Single objective optimization is derived as follow:

$$
\begin{aligned}
\min & \left(\sum_{i \in I} p_{i} \times C_{f i x i}+\sum_{i \in I, l \in K} Q_{i k} \times C_{\text {varik }}+\sum_{i \in I, l \in K, j \in J} S_{i k} \times C_{\text {trans }_{k}} \times Q_{\text {trans }_{\mathrm{j} k \mathrm{k}}} \times D_{i j}\right. \\
& \left.+\sum_{i \in I, l \in K, j \in J} Q_{\text {trans }_{\mathrm{jik}}} \times\left(D_{i j} / V\right) \times \text { Delay }_{\mathrm{c}_{k}}+\sum_{i \in I, l \in K, j \in J} Q_{\text {lack }_{\mathrm{jk}}} \times C_{\text {lack }_{k}}\right)
\end{aligned}
$$

While

$$
\text { s.t. }\left\{\begin{array}{c}
\sum_{k \in K} R_{i k} \times Q_{i k} \leq \operatorname{Cap}_{i}, i \in I \\
\sum_{j \in J} S_{i j k} \times Q_{\text {trans }_{i j k}} \leq Q_{i k}, i \in I, k \in K \\
\sum_{i \in I} S_{i j k} \times Q_{\text {trans }_{j i k}}+Q_{\text {lack }_{j k}} \leq Q_{i k}, i \in I, i \in J, k \in K \\
S_{i j k} \times D_{i j} \leq D_{\max i}, i \in I, i \in J, k \in K \\
S_{i j k} \leq R_{i k}, i \in I, i \in J, k \in K \\
P_{i}=\max \left\{R_{i k}, k \in K\right\}, i \in I \\
P_{i} \in\{0,1\}, i \in I \\
R_{i k} \in\{0,1\}, i \in I, k \in K \\
S_{i j k} \in\{0,1\}, i \in I, j \in J, k \in K \\
\geq 0, Q_{\text {trans }_{i j k}} \geq 0, Q_{\text {lack }_{j k}} \geq 0, i \in I, j \in J, k \in K
\end{array}\right.
$$


$C: C_{f i x i}$ represent the fixed cost while $\boldsymbol{C}_{\text {varik }}$ represents the fixed cost. $\boldsymbol{C}_{\boldsymbol{a p i}}$ is the ability of $i$.

$\boldsymbol{Q}_{\text {tran }}$ is the quantity of transport while $\boldsymbol{Q}_{\text {lack }}$ means the lack of resource. Other superscript represent points, which are omitted.

\subsection{Radiation Network model}

Since the transportation is also dynamic, we develop the radiation network to describe it. Single objective optimization is also established to build the network. That is

$$
\min \left(\sum_{i \in S E T} \sum_{j \in S E T} \sum_{m \in S E T} \sum_{n \in S E T} C_{k_{i j m n}} \times Q_{\text {trans }_{i j}} \times \text { flow }_{i j m n}\right)
$$

The constraint condition are

$$
\text { s.t. }\left\{\begin{array}{c}
\sum_{i \in S E T} h_{i} u b_{i}=N_{h u b s} \\
\forall i, j \in S E T: \sum_{m \in S E T} \sum_{n \in S E T} \text { flow }_{i j m n}=1 \\
\forall i, j, m, n \in S E T: \text { flow }_{i j m n} \leq h u b_{m} \\
\forall i, j, m, n \in S E T: \text { flow }_{i j m n} \leq h u b_{n} \\
\forall i \in S E T, \text { hub }_{i} \in\{0,1\} \\
\forall i, j, m, n \in S E T: \text { flow }_{i j m n} \in\{0,1\}
\end{array}\right.
$$

\section{Numerical calculation}

We use Xpress-mp to obtain the solution of multi-objective optimization. Germany, a federal parliamentary republic in West-Central Europe including 16 constituent states and covers an area of 357,021 square kilometers. By the administration, we decide the settlement points and supplement points. Through our model, we acquire the routes of transportation and the location of hubs. The result is shown in Figure 2.Since the radiation network we have obtained. It allows us find out the strategy of distribution and transportation.

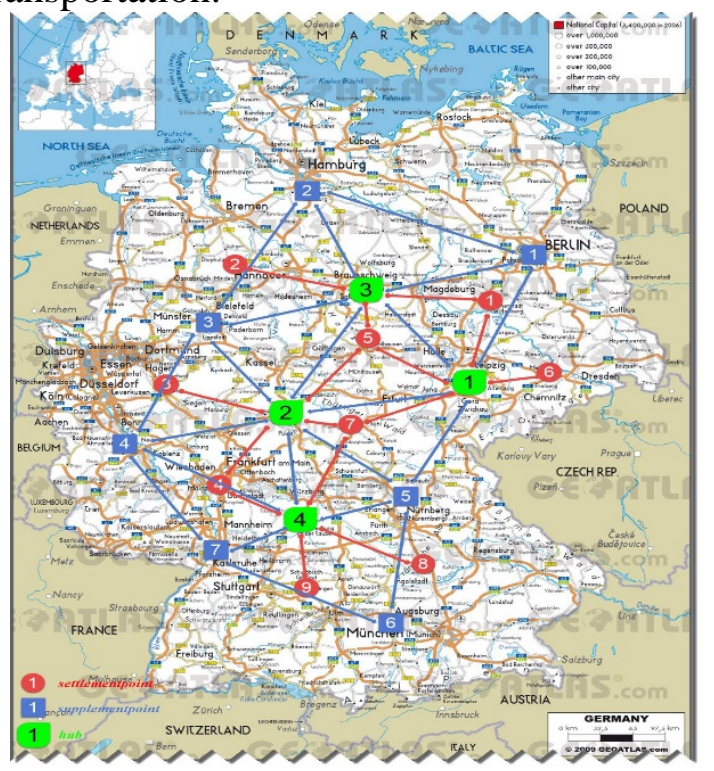

Fig 2. The locations and routes

We simulate the existence of refugees, dynamic changes because of time and environmental factors and final trends of the resettlement. The monthly supply of the resources and the relation to the demand figures. The possible situations are shown in Figure 3. 


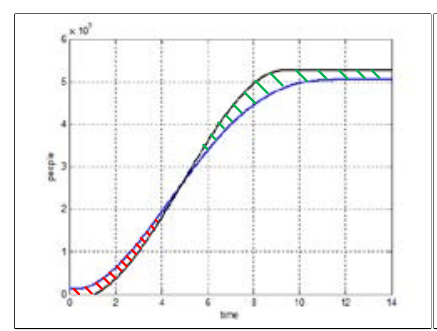

(a)

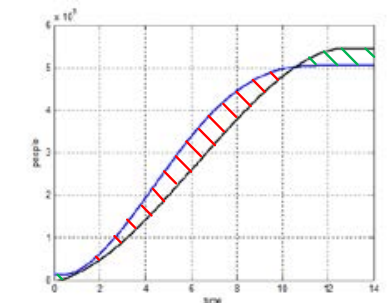

(b)

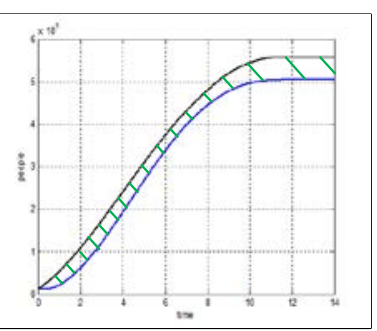

(c)

Fig. 3 relation between demand and supply

Red region represents the gap while green region represents the surplus.

(a) shows the ideal condition of the relation since the supply meets demand and is closed to it.

(b) shows the actual condition of the relation since the demand exceeding the supply. But with the specific policies, the need is satisfied finally.

(c) shows the ideal condition of the relation that the supply exceeding the demand. The death rate would decrease and requirement would be accomplished.

\section{References}

[1]Crina Grosan,Mihai Oltean. Adaptive representation for single objective optimization[J]. Soft Computing,2005,98:.

[2]S.K. Ong,S.H. Niu,A.Y.C. Nee. Improved Intelligent Water Drops Optimization for Single and Multiple Objective Job Shop Scheduling[J]. IFAC Proceedings Volumes,2013,469:. 